\title{
Membrana cerâmica assimétrica à base de argila para aplicação em processos de microfiltração: influência do tempo de deposição
}

\author{
(Asymmetric ceramic membrane based on clay for application in \\ microfiltration process: influence of time of deposition)
}

\author{
M. C. da Silva, H. de Lucena Lira, N. L. de Freitas \\ Departamento de Engenharia de Materiais, Universidade Federal de Campina Grande, Av. Aprígio Veloso 882, \\ Campina Grande, PB 58109-970. \\ mirelecsilva@hotmail.com,helio@dema.ufcg.edu.br,normanda@dema.ufcg.edu.br
}

\begin{abstract}
Resumo
A tecnologia de membranas vem sendo disseminada pelo mundo, em diversos setores onde existe a necessidade de separação de substancias, em diferentes escalas de dimensões e condições de temperatura e pressão. Neste sentido, as membranas cerâmicas vêm sendo exploradas para atuarem em ambientes onde as membranas poliméricas teriam restrições, como por exemplo, na presença de solventes orgânicos, altas pressões e temperaturas, etc. Diante disso, esse trabalho tem como objetivo a obtenção de membrana cerâmica assimétrica formada por uma camada filtrante à base de argila depositada sobre suporte poroso obtido com alumina comercial e bentonita. A camada ativa foi depositada por colagem em tempos de 10, 20 e 30 s para obtenção da membrana assimétrica. As massas cerâmicas para confecção do suporte e para obtenção da membrana foram caracerizadas por distrubuição granulométrica e microscopia eletrônica de varredura. O suporte poroso e a membrana assimétrica foram caracterizados por microscopia eletrônica de varredura, porosimetria por intrusão de mercúrio e foram realizadas análises de fluxo com água. Os resultados mostraram um suporte com diâmetro médio de $0,99 \mu \mathrm{m}$ e porosidade de $39,25 \%$. A membrana assimétrica foi obtida com sucesso, isenta de defeitos para tempo de colagem de $10 \mathrm{~s}$, atingindo uma espessura de camada depositada de $104 \mu \mathrm{m}$, diâmetro médio de poro de $0,25 \mu \mathrm{m}$ e porosidade de $35 \%$, sendo classificada, portanto, para aplicações em processos de microfiltração, com fluxo permeado estabilizado com água destilada em torno de $100 \mathrm{~L} / \mathrm{h} \cdot \mathrm{m}^{2}$.

Palavras-chave: membrana cerâmica, deposição, suporte, microfiltração.
\end{abstract}

\begin{abstract}
Membrane technology has been disseminated throughout the world, in several sectors where there is a need for separation of substances, in different scales of dimensions and conditions of temperature and pressure. In this way, ceramic membranes has been exploited to work in environments where the polymeric membrane have restriction, such as, in the presence of organic solvents, high pressures and temperatures, etc. So, the aim of this work is to obtain asymmetric ceramic membrane based on clay deposited on porous support obtained with commercial alumina. The active layer was deposited by slip casting with times of 10, 20 and $30 \mathrm{~s}$ to obtain the asymmetric membrane. The ceramics masses for the preparation of support and for the membrane were characterized by particle size distribution and scanning electron microscopy. The asymmetric porous support and the membrane were characterized by scanning electron microscopy, mercury intrusion porosimetry and water flow analyzes. The results showed a support with a mean diameter of $0.99 \mu \mathrm{m}$ and porosity of $39.25 \%$. The asymmetric membrane was successfully obtained, free of defects for bonding $10 \mathrm{~s}$, reaching a thickness of $104 \mu \mathrm{m}$, average pore diameter of $0.25 \mu \mathrm{m}$ and porosity of $35 \%$, thereby being considered for applications in the microfiltration process, with a stabilized distilled water flux permeate close to $100 \mathrm{~L} / \mathrm{h} . \mathrm{m}^{2}$.
\end{abstract}

Keywords: ceramic membrane, deposition, support, microfiltration.

\section{INTRODUÇÃO}

As membranas cerâmicas têm grande potencial para aplicação em que membranas poliméricas apresentam limitação e não podem ser usadas. Elas têm as vantagens de estabilidade térmica, de resistência a solventes e produtos químicos. Atualmente membranas cerâmicas de microfiltração e ultrafiltração estão disponíveis comercialmente e são aplicadas nas áreas de alimentos, bebidas e biotecnologia, representando cerca de $12 \%$ das membranas utilizadas nestas aplicações [1]. Além disso, outras vantagens dos materiais cerâmicos são a resistência mecânica e longa vida útil [2]. No entanto, os materiais cerâmicos e os processos envolvidos na obtenção de membranas ainda dificultam a competitividade destes produtos, fazendo com que os pesquisadores busquem materiais alternativos, tentando melhorar as propriedades apresentadas por esses materiais e minimizando os custos [3].

As membranas podem ser classificadas sob diferentes aspectos, de acordo com suas características. Uma dessas está relacionada à morfologia, podendo, por exemplo, ser porosa e assimétrica. Em geral, esse tipo de membrana é constituído por várias camadas (filmes extremamente finos contendo poros abertos) depositadas sobre um suporte poroso, de maneira que deixe passar o solvente da solução e retenha o 
soluto [4]. Os suportes porosos são usualmente fabricados por extrusão, tendo grandes tamanhos de poros em valores superiores a $1 \mu \mathrm{m}$ e com espessura da ordem de alguns milímetros e são responsáveis pela resistência mecânica. A camada de cobertura no suporte visa reduzir o tamanho dos poros e é chamada de camada de topo. Esta camada deve ser a mais fina possível e possuir o tamanho dos poros controlado para a separação especifica adequada. Portanto o tamanho dos poros de uma membrana assimétrica tem um gradiente a partir de um suporte poroso até uma camada de separação, com o objetivo de minimizar a resistência ao permeado através da membrana.

A deposição de camadas finas ou filmes é uma técnica em plena expansão, que pode ser realizada principalmente pelos processos de "sputtering", deposição química de vapor (CVD), "plasma spray", "flame spray", "dip coating" e "spin coating". Assim, é possível também depositar uma fina camada de outro material, formando uma nova membrana composta [4].

Para fabricação da membrana cerâmica assimétrica, um dos meios mais usados é o "dip coating". Para essa técnica é feita a preparação de uma dispersão aquosa, onde a aglomeração e a decantação são evitadas com o uso de dispersantes ou defloculantes. Para que a dispersão ocorra, é necessário que seja alcançada uma condição de equilíbrio entre as forças de atração e repulsão das partículas e a força de gravidade. A função do dispersante é evitar aglomerações de partículas, o que pode ser conseguido tornando-as eletricamente carregadas. Para isso modifica-se o $\mathrm{pH}$ da suspensão adicionando-se uma base $\left(\mathrm{OH}^{-}\right)$ou um ácido $\left(\mathrm{H}^{+}\right)$. Quando as cargas das partículas são idênticas há uma repulsão eletrostática entre elas e o sistema ficará disperso [5].

Para membranas cerâmicas compostas e/ou assimétricas além das características da pele, que é a parte ativa da membrana cerâmica, as características do suporte poroso também devem ser incluídas. A camada fina da membrana requer um substrato poroso que lhe confira uma resistência mecânica tal que permita a sua utilização em gradientes de pressão elevados, o que resultará num aumento no rendimento do processo.

Dessa forma, esse trabalho tem como objetivo a fabricação de membrana cerâmica assimétrica à base de argila e avaliar a influência do tempo de deposição da camada ativa sobre o suporte na morfologia das membranas com a finalidade de serem aplicadas em processos de microfiltração.

\section{MATERIAIS E MÉTODOS}

Para obtenção do suporte cerâmico foram utilizados alumina comercial (TAC) fornecida pela Treibacher Schleifmittel Brasil Ltda (90\%) e bentonita fornecida pela Bentonit União Nordeste (10\%). Para preparação da massa cerâmica para a obtenção da membrana cerâmica assimétrica foi utilizada uma argila plástica caulinítica contendo em sua estrutura mica, montmorilonita e sílica, resultado obtido através de difração de raios $\mathrm{X}$, não exibido no presente trabalho. A argila foi cedida pela empresa Armil Mineração Nordeste. Para preparação da dispersão foi utilizado o defloculante poliacrilato de amônia (Drew) e um antiespumante à base de silicone.

As matérias-primas foram misturadas manualmente a seco. Após total homogeneização desses materiais foi adicionado $10 \%$ em massa de água destilada, para atingir umidade adequada para a etapa de conformação. A massa obtida foi passada em peneira \#200 (abertura $0,075 \mathrm{~mm}$ ), segundo classificação da ABNT. Uma amostra desse material foi levada para estufa a $100{ }^{\circ} \mathrm{C} / 24 \mathrm{~h}$ para serem efetuadas as caracterizações por distribuição granulométrica em um analisador de tamanho de partículas por difração a laser, Cilas 1064 LD e por microscopia eletrônica de varredura (MEV) em um microscópio Shimadzu SSX-550.

A conformação dos suportes foi realizada no formato de discos por meio de prensagem uniaxial, $23 \mathrm{MPa}$, em um molde de aço inoxidável com $4 \mathrm{~cm}$ de diâmetro. Os suportes foram sinterizados em forno elétrico com temperatura máxima de $1200^{\circ} \mathrm{C}$ com os seguintes patamares de queima: temperatura inicial de $25 \mathrm{a} 700^{\circ} \mathrm{C}$ a uma taxa de aquecimento de $2{ }^{\circ} \mathrm{C} / \mathrm{min}$, permanecendo por $1 \mathrm{~h}$ e um segundo patamar de 700 a $1200{ }^{\circ} \mathrm{C}$ em uma taxa de aquecimento de $5{ }^{\circ} \mathrm{C} /$ mim permanecendo mais $1 \mathrm{~h}$. Foram realizadas as seguintes caracterizações no suporte sinterizado: microscopia eletrônica de varredura (MEV) e porosimetria por intrusão ao mercúrio em um porosímetro de mercúrio Autopore IV Micromeritics.

Para obtenção da membrana assimétrica foi preparada inicialmente uma dispersão, na proporção de (80-20)\% de água-massa dentro de um Becker de $250 \mathrm{~mL}$ com 0,7 $\mathrm{g}$ de defloculante. Este teor foi determinado pelo ensaio de viscosidade mínima, em viscosímetro Brookfield DVII+Pro. O valor mínimo de viscosidade foi de $12,00 \mathrm{cP}$. Uma gota de antiespumante foi adicionada à suspensão e em seguida colocada em um agitador Hamilton beach 930, a $17000 \mathrm{rpm}$, durante $30 \mathrm{~min}$. A dispersão ficou por mais 30 min sob vácuo, para eliminar possíveis bolhas de ar. Para preparação da membrana, foi feita a imersão do suporte sinterizado na dispersão dentro de um becker em tempos diferentes de 10, 20 e $30 \mathrm{~s}$. Uma das faces do suporte foi vedada com uma fita adesiva a fim de que a camada filtrante fosse formada em uma das faces do suporte.

As membranas assimétricas foram submetidas a etapa de sinterização em forno elétrico atingindo a temperatura máxima de $1200{ }^{\circ} \mathrm{C}$ seguindo os mesmos intervalos de queima do suporte poroso. Após sinterização as membranas foram caracterizadas por microscopia eletrônica de varredura (MEV) e por porosimetria de mercúrio. Foram realizadas análises de fluxo permeado pela membrana com água destilada a $25{ }^{\circ} \mathrm{C}$, em um sistema de laboratório. $\mathrm{O}$ cálculo para determinação do fluxo permeado em função do tempo foi feito utilizando-se a equação A:

$$
\mathrm{J}_{0}=\mathrm{m}_{\text {agua }} / \mathrm{t}_{\mathrm{c}} \cdot \mathrm{A}
$$

na qual $J_{0}$ é o fluxo permeado (L.h $\left./ \mathrm{m}^{2}\right), m_{\text {agua }}$ o volume de 
água (L) permeada durante o intervalo de tempo $t_{c}(\mathrm{~h}) \mathrm{da}$ coleta de água permeada pela membrana de área $\mathrm{A}\left(\mathrm{m}^{2}\right)$.

\section{RESULTADOS E DISCUSSÃO}

A Fig. 1 ilustra as curvas de distribuição granulométrica da massa cerâmica para confecção do suporte poroso e para a suspensão aquosa a ser depositada sobre o suporte para obtenção da membrana.

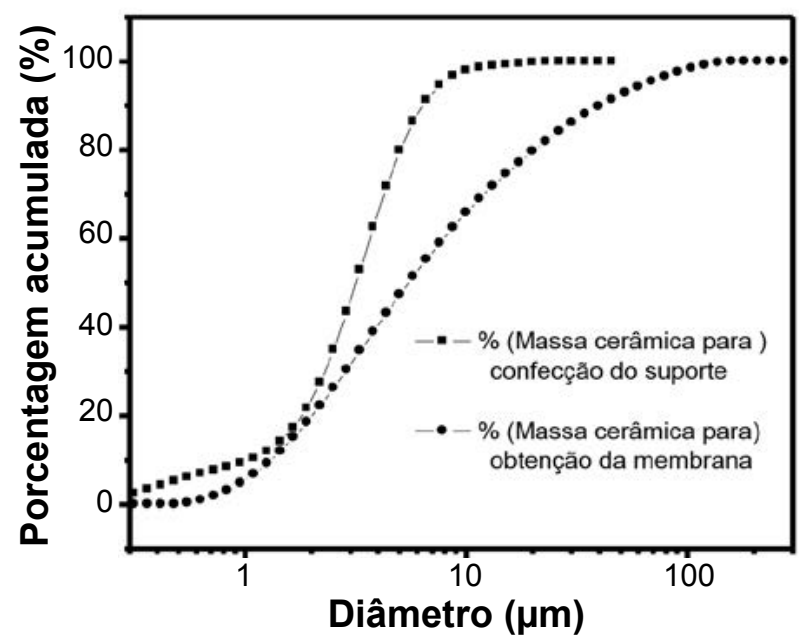

Figura 1: Distribuição granulométrica das partículas contidas na massa cerâmica para obtenção dos suportes cerâmicos e para obtenção da camada ativa da membrana assimétrica.

[Figure 1: Particle size distribution in the ceramic mass to obtain the ceramic supports and active layer of the asymmetric membrane.]

O percentual de acumulados para a massa do suporte mostra uma pequena quantidade de partículas em torno de $0,3 \mu \mathrm{m}$ relacionadas à argila presente na massa e uma maior concentração de partículas, numa faixa relativamente estreita de distribuição, com tamanho em torno de 3,3 $\mu \mathrm{m}$. Quanto aos valores dos diâmetros das partículas, $50 \%$ dos diâmetros médios apresentaram $3,17 \mu \mathrm{m}$ e um pequeno percentual de $10 \%$ valores de $1,04 \mu \mathrm{m}$. Quanto mais estreita a distribuição apresentada pela curva para diâmetro de partículas maior homogeneidade no que se refere à distribuição, tamanho e geometria dos poros do suporte cerâmico.

$\mathrm{Na}$ curva referente à massa para obtenção da suspensão, verifica-se que essa é constituída de partículas com tamanho que varia em sua maioria entre $1 \mathrm{a} 21 \mu \mathrm{m}$. O valor atingido para $50 \%$ dos diâmetros médios foi de $3,83 \mu \mathrm{m}$ e $10 \%$ de partículas com $1,16 \mu \mathrm{m}$.

Na obtenção de membranas cerâmicas assimétricas onde existe um gradiente de dimensão dos poros, o tamanho médio de partículas utilizadas na obtenção da camada ativa deve ser menor do que o tamanho médio de partículas utilizada na obtenção do suporte poroso para que a camada filtrante atinja poros de tamanho menores quando comparado aos poros do suporte que é responsável pela resistência mecânica da membrana, entretanto, nas curvas apresentadas na Fig. 1 ocorreu o inverso. Apesar de um desvio pequeno, o tamanho
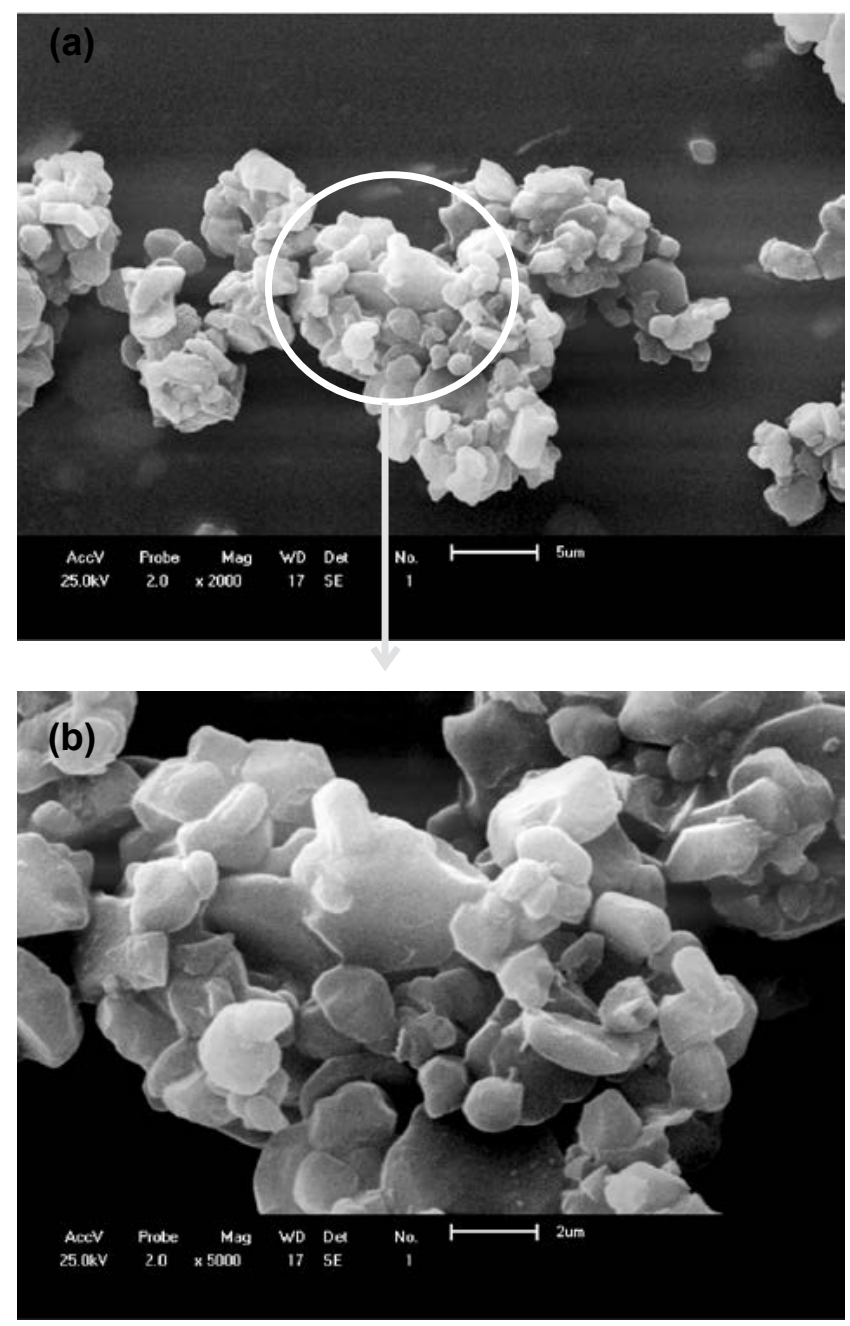

Figura 2: Micrografias obtidas em microscopia eletrônica de varredura da massa cerâmica preparada com alumina comercial e bentonita para obtenção dos suportes cerâmicos em aumentos de 2000x e 5000x.

[Figure 2: SEM micrographs of ceramic mass prepared with commercial alumina and bentonite to obtain the ceramic supports (top 2000x and bottom 5000x).]

médio de partícula utilizado para obtenção da camada ativa foi menor que o tamanho médio de partícula utilizada para o suporte. Essa divergência é justificada com base na Fig. 2 que exibe as micrografias da massa para confecção do suporte (a) e para obtenção da camada ativa (b).

As partículas de argila mostram dimensões em sua maior parte entre 1 e $2 \mu \mathrm{m}$; entretanto, verifica-se nitidamente a formação de aglomerados em empilhamento atingindo dimensão de aproximadamente $4 \mu \mathrm{m}$, o que na média para o cálculo da curva na Fig. 1 para dimensão dos aglomerados gerou o valor de $3,38 \mu \mathrm{m}$. Embora as partículas estejam aglomeradas em empilhamento, exibem uma distribuição uniforme no que diz respeito ao tamanho ilustrando geometria de placas. Visualmente os aglomerados apresentados também são constituídos de finas partículas. A Fig. 2a exibe as micrografias da massa cerâmica obtida com alumina e bentonita para obtenção dos suportes cerâmicos. As micrografias ilustram uma massa formada pela presença 

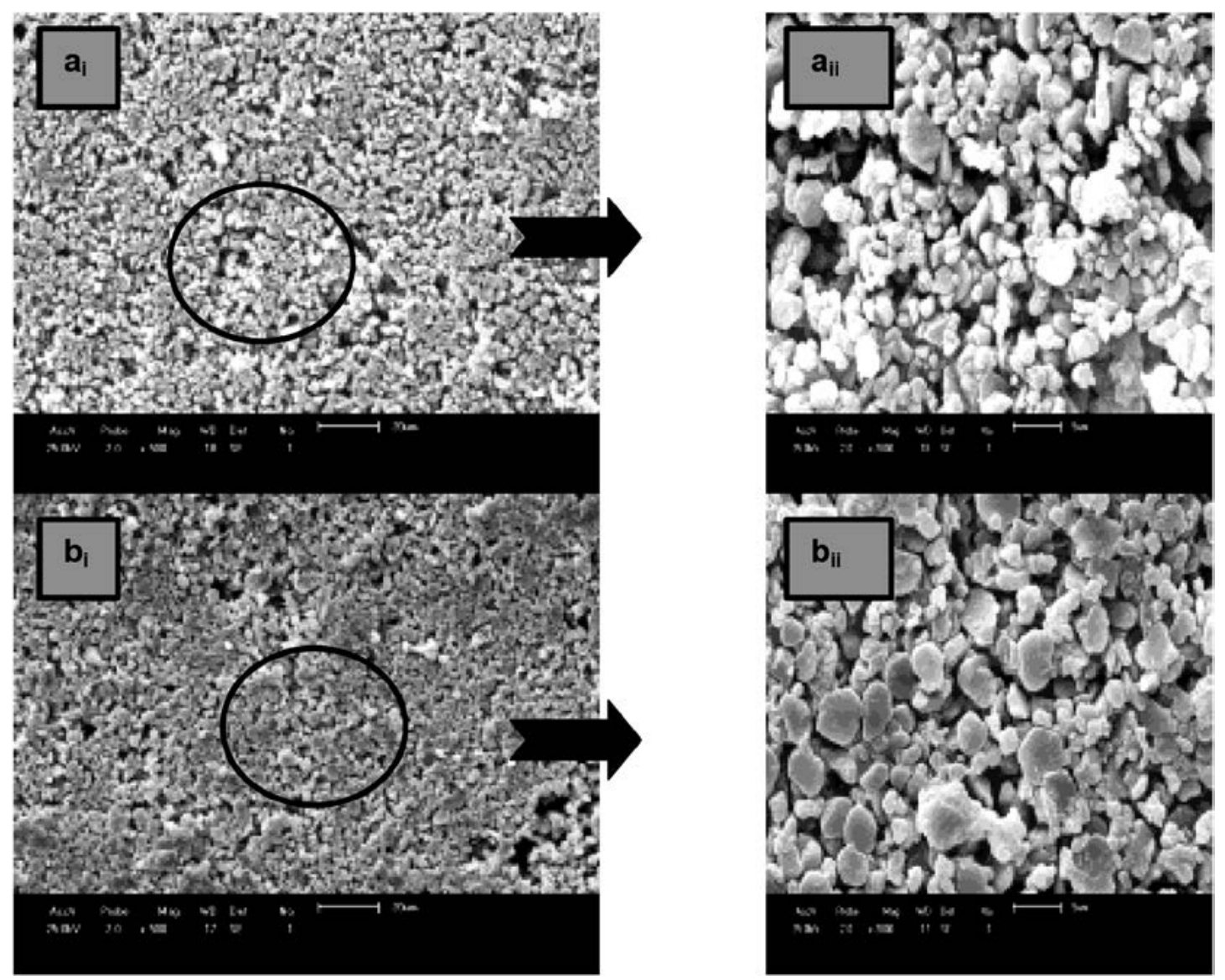

Figura 3: Micrografias obtidas em microscópio eletrônico de varredura do suporto cerâmico poroso obtidos em diferentes aumentos para superfície $\left(\mathrm{a}_{\mathrm{i}}=500 \mathrm{x} \mathrm{e}_{\mathrm{ii}}=2000 \mathrm{x}\right)$ e seção transfersal $\left(\mathrm{b}_{\mathrm{i}}=500 \mathrm{x}\right.$ e $\left.\mathrm{b}_{\mathrm{ii}}=2000 \mathrm{x}\right)$.

[Figure 3: SEM micrographs of the porous ceramic support abtained in different magnifications (a) surface $\left(a_{i}=500 x\right.$ and $\left.a_{i i}=2000 x\right)$ and cross-section $\left(b_{i}=500 x\right.$ and $\left.b_{i i}=2000 x\right)$.]

de partículas apresentando diferentes geometrias. Algumas exibem formato ligeiramente arredondado e outras alongadas, no entanto, todas apresentam dimensões abaixo de $5 \mu \mathrm{m}$. É possível observar aglomerados de partículas na forma de placas finas com formas irregulares, onde são características da morfologia típica da fase mais estável da alumina [6]. A geometria arredondada das partículas proporciona uma maior probabilidade de se obter suportes com maior homogeneidade de forma, tamanho e distribuição dos poros.

O suporte cerâmico apresentou uma retração de $12,3 \%$. Esta retração era esperada e está relacionada às diferentes etapas que a morfologia do material sofre durante o processo de sinterização. $\mathrm{O}$ aumento da temperatura de sinterização acelera o empescoçamento entre as partículas gerando uma maior densificação da peça e como conseqüência a retração das mesmas. Na composição preparada para obtenção do suporte foram utilizados alumina e bentonita, sendo que este último favorece a formação de fase líquida durante a etapa de sinterização, o que contribui para retração da peça.

A Fig. 3 expõe as micrografias obtidas da superfície (a) e a seção transversal (b) do suporte cerâmico. Há uma similaridade e homogeneidade de distribuição de tamanho de partículas para a seção transversal do suporte quando comparados com a superfície. É possível verificar a superfície do substrato ausente de trincas e/ou defeitos superficiais, bem como a presença de poros para todas as áreas analisadas e ligações entre os aglomerados de alumina. Nota-se a presença de espaços entre os grãos coalescidos, formando

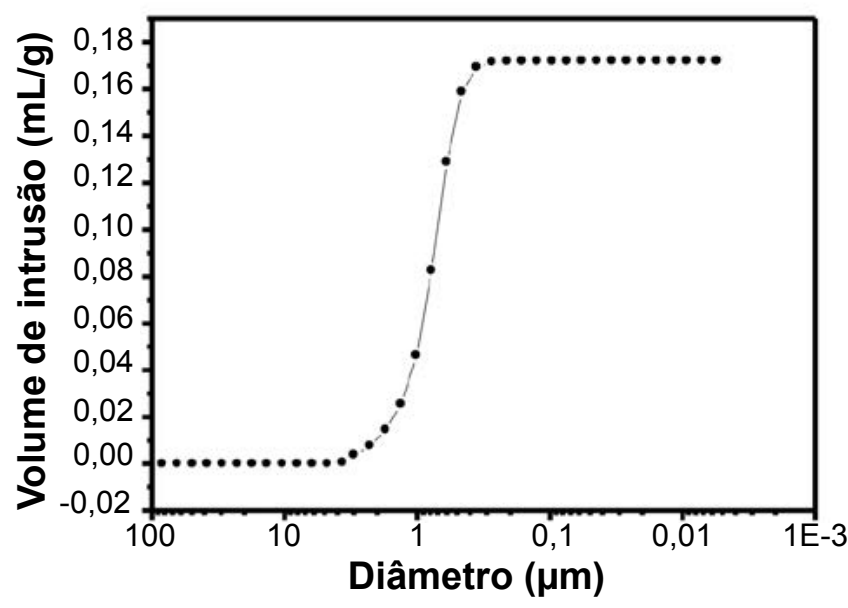

Figura 4: Curva da distribuição dos tamanhos dos poros no suporte cerâmico.

[Figure 4: Curve of the pore size distribution in the ceramic support.] 

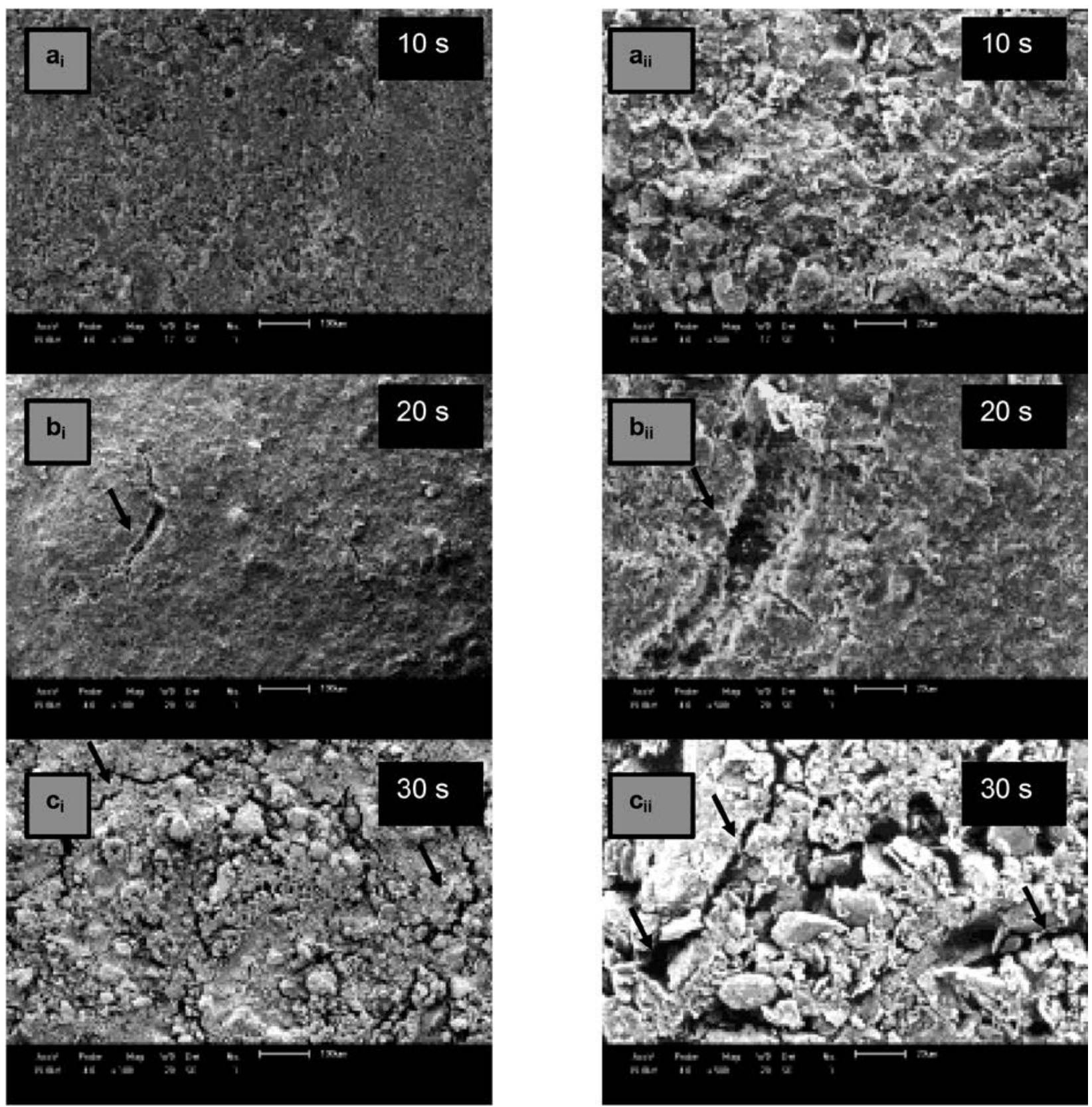

Figura 5: Micrografias obtidas em microscópio eletrônico de varredura da superfície das membranas assimétricas obtidas por deposição da barbotina para tempos de $10 \mathrm{~s}$ (a), $20 \mathrm{~s}$ (b) e 30 (c) em aumentos de $\left(\mathrm{a}_{\mathrm{i}}, \mathrm{b}_{\mathrm{i}}, \mathrm{e} \mathrm{c}_{\mathrm{i}}=100 \mathrm{x}\right.$ e $\mathrm{a}_{\mathrm{i}}, \mathrm{b}_{\mathrm{i}}$, e $\left.\mathrm{c}_{\mathrm{ii}}=500 \mathrm{x}\right)$.

[Figure 5: SEM micrographs related to the superface of asymmetric membranes obtained by depositing the slurry for times of $10 \mathrm{~s}$ (a), $20 \mathrm{~s}(\mathrm{~b}), 30 \mathrm{~s}(\mathrm{c})$ in increments of $\left(a_{i}, b_{i}\right.$, e $c_{i}=100 x$ and $a_{i j}, b_{i i}$, e $\left.c_{i i}=500 x\right)$.]

um material poroso.

As imagens possibilitam classificar os suportes quando ao tamanho dos diâmetros dos poros para processos de microfiltração uma vez que esses valores são inferiores a $2 \mu \mathrm{m}$, entretanto, as imagens não permitem estimar com precisão o tamanho dos poros. O tamanho de poro do suporte deve ser menor do que as partículas da camada a ser depositada e apresentar baixa rugosidade para evitar defeitos quando da formação da camada de topo da membrana [7].

A Fig. 4 exibe a curva correspondente a variação do diâmetro dos poros do suporte em função do volume de intrusão de mercúrio acumulado. O resultado obtido apresenta um suporte com diâmetro médio de poros de $0,99 \mu \mathrm{m}$ atingindo uma porosidade de $39,25 \%$. Com base na curva apresentada, percebe-se que a maioria dos valores referentes ao diâmetro médio dos poros estão na faixa de 1 a $0,4 \mu \mathrm{m}$. Quanto a porosidade apresentada, quanto maior esse valor, menor será a resistência ao fluxo do material que passa através da membrana. Esse valore está em concordância com a dimensão atingida pelo diâmetro de partícula da camada ativa $(3,83 \mu \mathrm{m})$, ou seja, a dimensão da partícula não deve ser menor do que o diâmetro médio dos poros do suporte a fim de evitar que partículas da barbotina não penetrem no suporte e diminua o fluxo permeado final da membrana. Alguns 


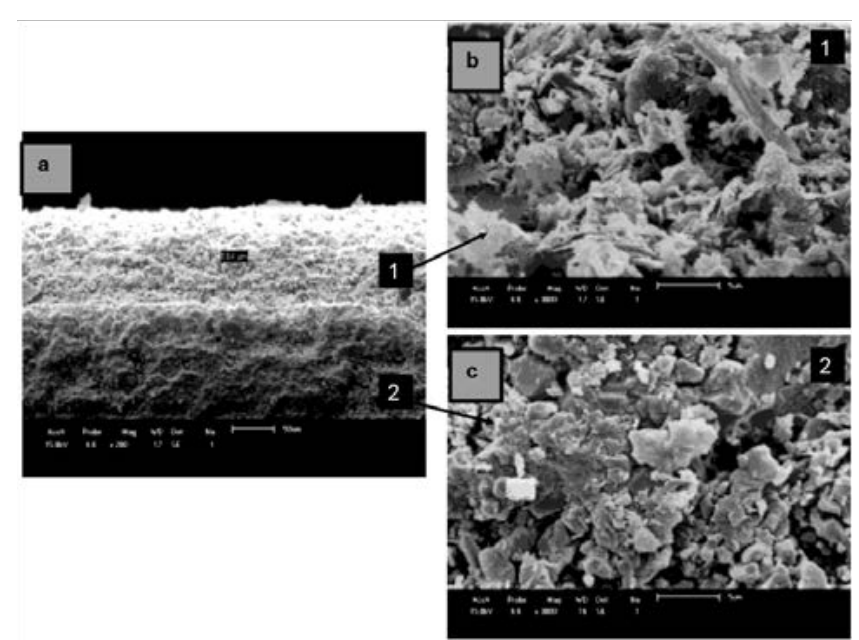

Figura 5: Micrografias obtidas em microscópio eletrônico de varredura da membrana cerâmica assimétrica obtida no tempo de $10 \mathrm{~s}$ de deposição da barbotina (a) interface, (b) camada de topo e suporte poroso.

[Figure 5: SEM micrographs of asymmetric ceramic membrane obtained at the time $10 \mathrm{~s}$ of deposition of the slip (a) interface, (b) top layer and (c) porous support.]

trabalhos recentes mostram a fabricação de membranas assimétricas utilizando-se alumina como matéria prima para confecção do suporte poroso. Foi utilizada alumina para suportes atingindo poros de $0,2 \mu \mathrm{m}$ para fabricação de membrana de paládio [8] e foi confeccionado suporte de alumina para fabricação de membrana assimétrica de $\mathrm{TiO}_{2}$ para aplicações em fotocatálise [9].

A porosidade final da membrana e o tamanho médio de poros dependem, entre outros, do material, da granulometria do pó, da pressão aplicada no molde e da temperatura de sinterização, ou seja, a complexa morfologia para montagem

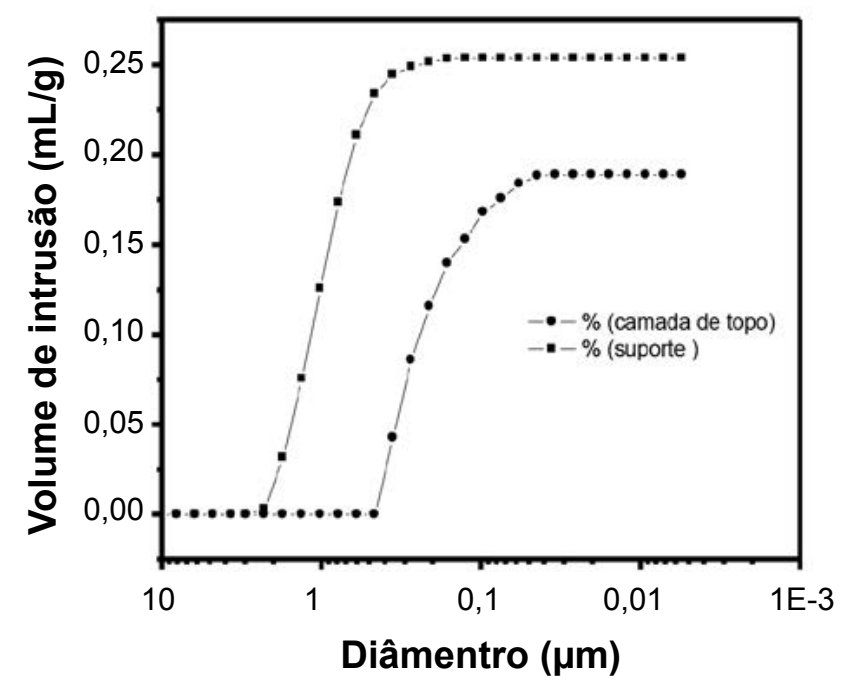

Figura 7: Curvas da variação do diâmetro médio dos poros em função do volume de intrusão de mercúrio acumulado para o suporte isolado e para a camada de topo.

[Figure 7: Curves of the variation of the average pore diameter as a function of cumulative intrusion volume of mercury to isolated support and to top layer.] da membrana assimétrica não pode ser justificada com base em uma caracterização apenas [4].

A Fig. 5 mostra as micrografias da superfície das membranas cerâmicas assimétricas obtidas em tempos de deposição da barbotina de $10 \mathrm{~s}$ (a), 20 s (b) e $30 \mathrm{~s} \mathrm{(c).}$ Pode-se observar que a variação do tempo de deposição das barbotinas sobre o suporte é suficiente para alterar a morfologia da membrana assimétrica. $\mathrm{O}$ aumento no tempo provocou defeitos, tais como, fissuras e falta de aderência da camada da superfície da membrana. O melhor resultado foi apresentado pela membrana obtida por $10 \mathrm{~s}$, a qual não apresentou defeitos e com uma camada distribuída de forma homogênea. Para a membrana obtida em 20 s verificamse defeitos, como fissuras. E para a membrana obtida em $30 \mathrm{~s}$, além de inúmeros defeitos, é possível observar que não houve uma boa adesão da camada sobre o suporte poroso. Dessa forma, as membranas obtidas em $10 \mathrm{~s}$ foram escolhidas para as próximas caracterizações.

A Fig. 6 apresenta a micrografia da seção transversal da membrana cerâmica assimétrica obtida em $10 \mathrm{~s}$ de deposição da barbotina para as seções (a) interface, (b) camada de topo e (c) suporte poroso. Com base na Fig. 8 pode-se observar a obtenção de uma membrana assimétrica mostrando a interface entre o suporte (2) e a camada ativa (1). A espessura desta camada (1) foi estimada em $104 \mu \mathrm{m}$, depositada sobre o suporte poroso de alumina (2). Há a formação de uma camada homogênea, presença de poros, interfaces regulares e a ausência de trincas ou defeitos superficiais, além de aglomerados de partículas abaixo de $5 \mu \mathrm{m}$. Pode-se notar facilmente diferenças morfológicas apresentada por ambas camadas. A camada (1) é constituída de partículas com morfologia de placas, característico das argilas, enquanto que o suporte poroso (2) exibe partículas mais grosseiras, constituído de aglomerados fortemente ligados e sinterizados.

A Fig. 7 exibe as curvas referentes à distribuição do

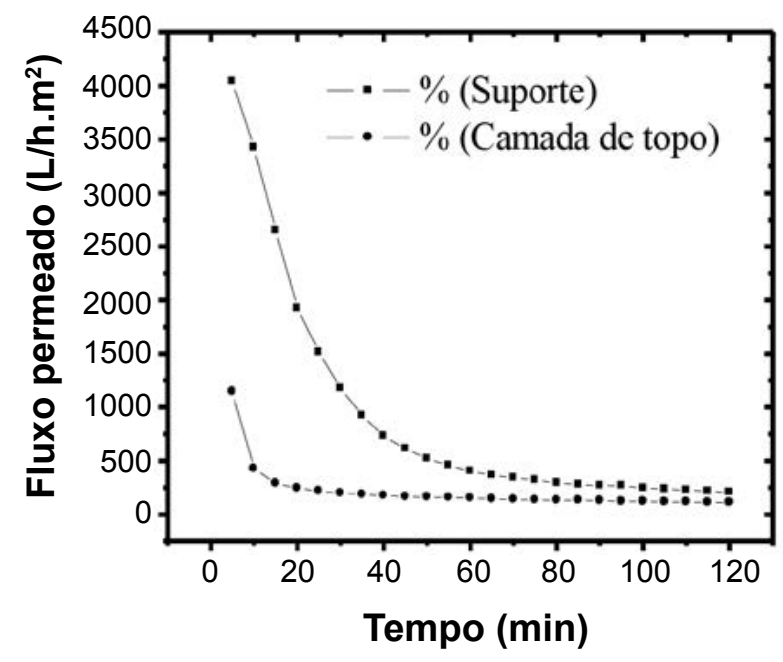

Figura 8: Fluxo permeado com água destilada pelo suporte poroso e pela membrana assimétrica.

[Figure 8: Permeate flow with distilled water for the porous support and asymmetric membrane.] 
tamanho dos poros para o suporte poroso, comparando-o com o da camada de topo da membrana.

Deve-se salientar que os dados obtidos para a camada de topo da membrana foram obtidos em um corpo de prova na forma de disco obtido por colagem, sinterizado nas mesmas condições da membrana assimétrica; isto foi necessário em função do pequeno volume de poros desta fina camada depositada e que ficaria imperceptível de se observar na membrana integral. Observa-se que a membrana produzida apresenta a maioria dos poros com diâmetro variando em torno de 0,4 e $0,04 \mu \mathrm{m}$, como pode ser observado na inclinação da curva para essa faixa e diâmetro médio de $0,25 \mu \mathrm{m}$, com porosidade de $35 \%$, enquanto que o suporte apresentou uma maior variação, em $\sim 3,0 \mu \mathrm{m}$ e porosidade de $39,25 \%$. Pode-se verificar que tanto o suporte quanto a membrana apresentaram uma estrutura unimodal e uma distribuição relativamente estreita de poros.

Os resultados dos fluxos permeados pela membrana e pelo suporte, com água destilada estão ilustrados nas curvas apresentadas na Fig. 8. O comportamento do fluxo permeado durante o ensaio foi semelhante para o suporte poroso e membrana assimétrica, os dois apresentaram um fluxo bastante elevado no inicio, em torno de $4000 \mathrm{~L} / \mathrm{h} . \mathrm{m}^{2}$ e $1200 \mathrm{~L} / \mathrm{h} . \mathrm{m}^{2}$, respectivamente e após $10 \mathrm{~min}$ de ensaio apresentaram uma queda brusca, estabilizando em torno de 205,0 e 107,0 L/h.m ${ }^{2}$ para o suporte e a membrana, respectivamente. Essa queda no fluxo deve-se a hidratação da membrana que se estabiliza após um tempo de aproximadamente $10 \mathrm{~min}$.

\section{CONCLUSÕES}

A utilização de matérias primas naturais é satisfatória para obtenção de membranas cerâmicas assimétricas quando depositadas no tempo de $10 \mathrm{~s}$ sobre suporte poroso de alumina comercial. As membranas produzidas para esse intervalo de tempo apresentaram-se isenta de defeitos, com média de diâmetro de poro de $0,25 \mu \mathrm{m}$ atingindo porosidade de $35 \%$, sendo classificadas, portanto, para aplicações em processos de microfiltração, atingindo fluxo permeado estabilizado para água destilada de 107,0 L/h.m².

\section{AGRADECIMENTOS}

À CAPES pelo suporte financeiro, ao Laboratório de Síntese de Materiais Cerâmicos (Labsmac) e ao Laboratório de Referencia em Dessalinização (Labdes), ambos da Universidade Federal de Campina Grande (UFCG), pelo uso de suas instalações e apoio à pesquisa.

\section{REFERÊNCIAS}

[1] R. R. Bhave, Inorganic Membranes: Synthesis, Characterization, and Applications, Van Nostrand Reinhold, New York, EUA (1991).

[2] H. P. Hsieh, Inorganic Membranes for Separation and Reaction, Elsevier, Amsterdam (1996).

[3] D. F. Stamatialis, B. J. Papenburg, M. Gironés, S. Saiful, S. N. M. Bettahalli, S. Schmitmeier, M. Wessling. J. Membrane Sci. 308, 1-2 (2008) 1-34.

[4] A. C. Habert, C. P. Borges, R. Nobrega, Processos de Separação por Membranas, E-Papers Serviços Editoriais, Rio de Janeiro, RJ (2006) p. 20.

[5] J. Catafesta, R. Andreola, C. A. Perottoni, J. E. Zorzi, Cerâmica 53, 325 (2007) 29-34.

[6] L. S. Neiva, A. C. F. M. Costa, H. M. C. Andrade, L. Gama, Cerâmica 58, 346 (2012) 186-194.

[7] A. J. Burggraaf, L. Cof, Membrane Sci. Technol. 4, 1 (1996) 1-20.

[8] X. Hu, J. Yu, J. Song, X. Wangb, Y. Huang, Int. J. Hydrogen Energy 36, 24 (2011) 15794-15802.

[9] R. Goei, T. T. Lim. Ceram. Int. 40, 5 (2014) 6747-6757. (Rec. 18/04/2014, Rev. 06/06/2014, Ac. 28/06/2014) 